\title{
Clinicopathological Features and Metastatic Pattern of Triple Negative Breast Cancer Among Female Patients at a Tertiary Care Hospital
}

fatma khinaifis Al thoubaity

king abdulaziz university hospital, Saudi Arabia

\begin{abstract}
:
Background: Breast cancer $(\mathrm{BC})$ is a heterogeneous disease based upon the status of three receptors. Triple negative $\mathrm{BC}$ (TNBC), a BC subtype, lacks the expression of estrogen and progesterone receptors as well as expression and amplification of HER2/neu and It appears more frequently in younger age group.

Objectives: analyze the clinicopathological features and metastatic rate of TNBC in Saudi Arabian women.

Methods: A seven-year retrospective study was conducted at King Abdulaziz University Hospital in Jeddah, Kingdom of Saudi Arabia. All females with TNBC diagnosed between January 1, 2010, and June 30, 2017, were enrolled. Demographic and clinical data were then obtained from medical records.

Results: Among 1209 BC patients, TNBC cases were $152(17 \%)$. The mean age at the time of diagnosis was 48.82 years and the mean body mass index (BMI) was $29.3 \mathrm{~kg} / \mathrm{m} 2$. The majority of the patients were obese. The most common symptoms were palpable axillary tail mass $(17.1 \%)$, pain (16.4\%), and nipple changes (14.5\%). In our study, only $3.3 \%$ of patients
\end{abstract}


experienced nipple discharge and 9.9\% had a family history of $\mathrm{BC}$. The average size of the tumor was $3.9 \mathrm{~cm}$. The mortality rate was significant $(21.7 \%)$. The recurrence and metastasis rates were $20.4 \%$ and $33.6 \%$, respectively.

Conclusion: TNBC development was not significantly associated with the age, tumor and BMI. In addition, the majority of TNBC patients were obese, suggesting that weight of the patients is a significant factor associated with the development of TNBC .Recurrence and metastatic rate are high.

Keywords:breast cancer, triple negative, metastases, recurrence.

\section{Advances in Knowledge:}

Triple negative breast cancer has high rate of metastasis and reoccurrence with poor prognosis which warrant earlier and more frequent screening for metastasis in the course of the disease.

\section{Application to patient care:}

It is important to approach breast cancer upon the molecular subtype as it's a heterogenous disease with each subtype behaving differently. Better understanding of each molecular subtype will further help targeted approach.

\section{Introduction}

Breast cancer (BC) is one of the most commonly diagnosed cancer among woman. It affects more than 1.5 million women each year globally and is the second leading cause of death after lung cancer. ${ }^{1}$ The global standardized mortality rate of $\mathrm{BC}$ is $12.9 / 1,000,000 .{ }^{2}$ Moreover, the incidence of $\mathrm{BC}$ is increasing in nearly every region worldwide. ${ }^{1}$ In Saudi Arabia, BC ranks number one among the cancers in Saudi women and its incidence is increasing annually .In 2010, 1473 cases of BC were reported, with an age-standardized incidence rate of $24.9 / 100,000 .^{3} \mathrm{BC}$ affects the younger age group and is detected at a more advanced stage. ${ }^{4}$ Therefore, more diagnostic methods for detecting $\mathrm{BC}$ are required to be developed for 
providing a timely treatment, which could save many lives. $\mathrm{BC}$ is a heterogeneous disease characterized by different molecular subtypes that are classified based on the expression of specific receptors, which are detected by immunohistochemistry (IHC). These receptors include estrogen receptor (ER), progesterone receptor (PR) and human epidermal growth factor 2 receptor (HER2). Clinicians rely on the expression status of these receptors for predicting the behavior and outcome of $\mathrm{BC} .^{5}$ Triple negative $\mathrm{BC}$ (TNBC) is a rare, aggressive subtype of $\mathrm{BC}$, which lacks the expression of ER, PR, and HER2. A vast majority of these tumors are less-differentiated carcinomas, belonging to the basal-like subgroup. They are characterized by a frequent local recurrence, organ metastases,${ }^{6}$ and poor outcomes compared with other cancer subtypes. ${ }^{7-14}$ They are usually associated with the occurrence of hereditary BCs, which are caused by BRCA1 and rarely BRCA2 gene mutations. ${ }^{6}$ However, the current drugs and hormone therapies that target HER2 are unable to treat BC. Hence, more in-depth studies on the presentation patterns of this BC subtype are required to be conducted. In this study we explored the incidence, clinicopathological features, recurrence, and metastatic features of TNBC among female patients diagnosed at King Abdulaziz University Hospital (KAUH), Jeddah, KSA.

\section{Materials and Methods}

\section{Study population}

We retrospectively investigated the clinicopathological features and metastatic pattern of TNBC in female patients at the KAUH in Jeddah, Saudi Arabia, in 2017. The study was approved by the Institutional Review Board of the KAUH. Electronic medical records of all female patients diagnosed with primary BC from January 1, 2010 to June 30, 2017 at KAUH were extracted from the KAUH phoenix system using the International Classification of Diseases, tenth revision (ICD-10) codes (C50.011-D05.82).A total of 1209 primary BC female patients were identified based on the histopathology reports. Breast cancer lacking HER2, ER, and PR are said to be HER2-, ER-, and PR-negative (triple negative breast cancer). Thus, all patients were analyzed for these parameters.

\section{ER/PR staining}

Immunohistochemistry data for all patients were analyzed to determine ER and PR expression status Testing for ER and PR receptors is the standard procedure for the diagnosis of BC. These tests aid in determination of a patient's risk of cancer recurrence and whether 
the cancer can be treated with hormone therapy. ER or PR are considered positive if there is at least 1 percent $(\geq 1 \%)$ of immunoreactive tumor cell nuclei present and a sample is considered negative if less than 1 or 0 percent $(<1 \%)$ of tumor cell nuclei are immunoreactive.

\section{Her2/neu status}

IHC data of all patients were analyzed to determine HER2/neu expression status".HER2/neu results guide in making the best possible treatment decision. A scoring system is used to report the results of Her2/neu. A score of 0 to $1+$ is considered negative and that of $3+$ is considered positive. However, in the current study, a score of 2+ was regarded as negative unless it was verified to be positive by fluorescent in situ hybridization.

\section{Data collection}

Data regarding patients' demographics, age at diagnosis, site, clinical presentation, histopathological feature, history of metastasis, recurrence, and surgical interventions were extracted from the medical records. Data were stored in Microsoft Excel 2014.

\section{Statistical analysis}

\section{Tests of association (correlation)}

To analyze the associations between different variables, appropriate statistical tests were used. Three different types of variables (i.e., nominal, ordinal, and scale/continuous variables) were analyzed using a specific statistical test for each type. The means and standard deviation were calculated for each variable without missing values. The chi-square test was used for variables with significant "correlation. Nominal variables were analyzed using the Phi, Cramer's V, and Lambda tests. Ordinal variables were analyzed using the Gamma test. "Scale/continuous variables were analyzed using the correlations test of significance and independent sample t-test". In test of significance, correlation coefficients significant at 0.05 are denoted by a single asterisk and those significant at 0.01 are denoted by two asterisks.

\section{Ethical approval:}

There were minimal ethical implications and issues since it is a retrospective study. Patient identity and confidentiality were protected by assigning each patient a specific serial number. Moreover, no one except the investigating research team accessed the patients' records. We obtained prior approval from the Institutional Review Board since a consent form was not applicable to our study. 


\section{Results}

A total of 1209 patients were analyzed for the status of receptors using IHC data from pathology reports. Of these, only 152 (17\%) patients had TNBC (ER-, PR-, and Her2-). The most frequent subtype was luminal A (ER+, Her2-, and G1/2) with 406 (34\%) patients, followed by HER-enriched (ER- and/or PR - and Her2+) with 169 (14\%) patients, triple positive BC with $124(10 \%)$ patients, and luminal B with $81(7 \%)$ patients. Hence, all the analyses were performed in 152 TNBC patients.

\section{Epidemiological features of Triple negative breast cancer (TNBC):}

Patient distribution by nationality revealed that non-Saudi patients accounted for $58.6 \%$ and Saudi patients accounted for $41.4 \%$. The mean (SD) age of patients was $48.82( \pm 12.034)$ years (range: $23-85$ years). Patients were grouped based on their age into the following five groups: Group 1 (23-30 years) comprising 7.2\% patients; Group 2 (31-40 years) comprising 17.1\%; Group 3(41-50 years) comprising 32.9\%; Group 4 (51-59 years) comprising 28.9\%; and Group 5( $\geq 60$ years) comprising $13.8 \%$ patients. approximately $33 \%$ of the patients belonged to Group 3 and 30\% to Group 4.

The mean (SD) of the body mass index (BMI) was $29.3( \pm 6.43)$ (range: 17.30-54.68). Majority of TNBC patients $(n=64,42.1 \%)$ were obese, followed by $(n=44,28.9 \%)$ were overweight. Normal weight $(\mathrm{n}=42,27.6 \%)$ with only 2 patients being underweight at time of the diagnosis $1.3 \%$.

BMI by age group shows that the majority of the obese patients were among the age group from 40 to 50 years, followed by the age group more than 60 years old.

And the majority of the overweight patients were in the age group from 30 to 40 years old. While the majority of normal weight patients were in the age group from 20 to 30 years old. Tables. 1 illustrates the BMI distribution in different age groups.

\begin{tabular}{|c|c|c|c|c|c|}
\hline \multicolumn{2}{|c|}{ Table.1: BMI distribution in different age groups } \\
\hline \multirow{2}{|c|}{$\begin{array}{c}\text { BMI categories } \\
\text { Weight }(\mathrm{kg}) / \text { Highest2 }(\mathrm{m} 2)\end{array}$} & $\begin{array}{c}\text { Underweight } \\
<18.5\end{array}$ & $\begin{array}{c}\text { Normal weight } \\
18.5-24.9\end{array}$ & $\begin{array}{c}\text { Overweight } \\
25-29.9\end{array}$ & $\begin{array}{c}\text { Obese } \\
>30\end{array}$ \\
\hline \multirow{3}{*}{$\begin{array}{c}\text { Age } \\
\text { group }\end{array}$} & $23-30$ years & $0 \%$ & $36.4 \%$ & $36.4 \%$ & $27.3 \%$ \\
\cline { 2 - 3 } & $30-40$ years & $0 \%$ & $26.9 \%$ & $42.3 \%$ & $30.8 \%$ \\
\hline & $40-50$ years & $2 \%$ & $24 \%$ & $24 \%$ & $50 \%$ \\
\hline
\end{tabular}




\begin{tabular}{|l|c|c|c|c|c|}
\hline $50-60$ years & $2.3 \%$ & $31.8 \%$ & $25 \%$ & $40.9 \%$ \\
\hline & $>60$ years & $0 \%$ & $23.8 \%$ & $28.6 \%$ & $47.6 \%$ \\
\hline
\end{tabular}

All TNBC patients were asked about family history of Breast cancer in general. Only 9.9\% $(n=15)$ had positive family history whether in first-degree or second-degree relatives and majority $90.1 \%(n=137)$ have no family history of $\mathrm{BC}$ at all. In regard to the smoking status, $98 \%$ of the patients are not smokers compared to $2 \%$ only smokers. Symptom analysis revealed that $16.4 \%$ of the patients had breast pain, $3.3 \%$ had nipple discharge, $17.1 \%$ had palpable axillary lymph node, $14.5 \%$ experienced changes in their nipples.

\section{Histopathological features of Triple negative breast cancer (TNBC):}

The tumors had a median size of $3.92 \mathrm{~cm}$. with the largest being $18 \mathrm{~cm}$ in size and the smallest being $0.20 \mathrm{~cm}$ in size. The most common pathological type was invasive ductal carcinoma $(n=137,90.1 \%)$ with a ductal carcinoma in situ component co-existing with invasive $\mathrm{BC}$ in $16.4 \%$ of the time $(\mathrm{n}=25)$. At presentation, Most of the tumors had poorly differentiated histological grade. $3(\mathrm{n}=94,62.3 \%)$, Histological grade.2 for moderately differentiated tumors was found in $27.8 \%$ of the biopsies $(n=42)$. Histological grade1 for well-differentiated tumors was found in $9.9 \%(n=15)$.

The biopsy margin was negative in $86.8 \%$ of the patients and positive in $11.2 \%$ of the patients. Lymph node involvement. In regard to the lymph nodes status, involvement happened $33.6 \%(n=51)$ and the average number of lymph nodes involved per patients who had positive lymph node deposition was 2.52 (range: $0-40$ ).

\section{Recurrence rate:}

Over a median follow up period of 17 months, BC recurrence happened in $20.4 \%$ of the cases $(n=31)$. Based on age groups, Group 1 (23-30 years) had the highest $(27.3 \%)$ the recurrence rate followed by Group 4 (51-59 years) and Group 5( $\geq 60$ years) had the lowest recurrence rate $(14.3 \%)$. Further, based on nationality, the recurrence rate was higher among non-Saudi patients $(22.5 \%)$ than among Saudi patients (17.5\%).Table.2 represents the Recurrence rate of TNBC in different age groups. 


\begin{tabular}{|c|c|c|}
\hline \multicolumn{2}{|c|}{ Table.2: Recurrence rate of TNBC in different age groups } \\
\hline Age group & No & Yes \\
\hline $23-30$ years & $72.7 \%$ & $27.3 \%$ \\
\hline $30-40$ years & $80.8 \%$ & $19.2 \%$ \\
\hline $40-50$ years & $80.0 \%$ & $20.0 \%$ \\
\hline $50-60$ years & $77.3 \%$ & $22.7 \%$ \\
\hline$>60 \quad$ years & $85.7 \%$ & $14.3 \%$ \\
\hline
\end{tabular}

\section{Metastasis rate:}

Over a median follow up period of 17 months, Distant metastasis was noted in $33.6 \%$ of the patients. Based on nationality, the metastasis rate was higher among non-Saudi patients (39.3\%) than among Saudi patients (25.4\%), and based on age groups, a negative relationship was noted between the age group and metastasis rate. Group 1 (23-30 years) had the highest metastasis rate $(45.5 \%)$, whereas and Group 5( $\geq 60$ years) had the lowest $(28.6 \%)$.The results revealed that $14.5 \%$ had bone metastases ,lung metastasis were $22.4 \%$ and liver metastasis were $15.8 \%$,brain metastasis were $3.3 \%$ and skin metastasis were $2 \%$. Table. 3 shows distant metastasis rate in patients of different of nationality and age groups.

Table 3. Distant metastasis rate in patients of different of nationality and age groups

\begin{tabular}{|c|c|c|c|}
\hline \multicolumn{2}{|c|}{ Distant metastasis } & No & Yes \\
\hline \multirow{3}{*}{ Nationality } & Saudi & $74.6 \%$ & $25.4 \%$ \\
\cline { 2 - 4 } & Non-Saudi & $60.7 \%$ & $39.3 \%$ \\
\hline \multirow{3}{*}{ Age group } & $23-30$ years & $54.5 \%$ & $45.5 \%$ \\
\cline { 2 - 4 } & $30-40$ years & $61.5 \%$ & $38.5 \%$ \\
\cline { 2 - 4 } & $40-50$ years & $66.0 \%$ & $34.0 \%$ \\
\cline { 2 - 4 } & $50-60$ years & $70.5 \%$ & $29.5 \%$ \\
\hline & $>60$ years & $71.4 \%$ & $28.6 \%$ \\
\hline
\end{tabular}




\section{Tests of association:}

The result of chi square test of association shows that there is no significant association between patient's age at diagnosis and systemic metastasis nor axillary lymph nodes status, $\mathrm{P}$-value $=0.82, \mathrm{P}$-value $=0.54$ respectively.

Further, A Pearson correlation data analysis revealed that the patient's age at diagnosis (mean= 48.8, $\mathrm{SD}= \pm 12$ ) and tumor size (mean= 3.9, $\mathrm{SD}= \pm 2.4$ ) was not significantly correlated $\mathrm{r}=-0.07$, Neither was BMI (mean= 29.3, $\mathrm{SD}= \pm 6.4$ ) and tumor size has any significant linear relationship $r=-0.04$. Moreover, chi square testing shows no significant association between BMI and histological grade of the tumor, $\mathrm{P}$-value $=0.26$.

\section{Therapeutic approaches:}

A total of $93.3 \%$ of the cases had surgery. The most common surgical intervention performed for TNBC was modified radical mastectomy rather than conservative breast therapy. Of those who underwent surgery, $6.5 \%$ had postoperative complications as follows: wound infection $3.9 \%$ and seroma $2.6 \%$ Of these, $18.3 \%$ had to go for another surgical intervention in the same breast. Over a median follow up period of 17 months, the mortality rate was $21.56 \%$ $(\mathrm{n}=33)$. Table $\underline{4}$ summarizes the attempted interventions.

\begin{tabular}{|c|c|}
\hline Table.4 Type of intervention & No. of subject $(\%)(n=152)$ \\
\hline primary surgical intervention & $142(93.3 \%)$ \\
\hline Mastectomy with sentinel lymph node biopsy & $13(8.6 \%)$ \\
\hline Modified radical mastectomy & $64(42 \%)$ \\
\hline Lumpectomy with axillary dissection & $39(25.7 \%)$ \\
\hline Lumpectomy with sentinel lymph node biopsy & $26(17 \%)$ \\
\hline Secondary surgical intervention & $28(18.3 \%)$ \\
\hline Reconstructive/debridement & $7(4.6 \%)$ \\
\hline Re-lumpectomy & $6(3.9 \%)$ \\
\hline Re-mastectomy & $15(9.8 \%)$ \\
\hline
\end{tabular}




\begin{tabular}{|c|c|}
\hline Radiotherapy & $93(60.8 \%)$ \\
\hline Adjuvant chemotherapy & $97(63.4 \%)$ \\
\hline Neoadjuvant chemotherapy & $50(32.7 \%)$ \\
\hline
\end{tabular}

\section{Discussion}

In developing countries, breast cancer is common with high mortality rate. $\mathrm{BC}$ is one of the major causes of death in women in many countries. ${ }^{15}$ Approximately $38 \%$ of deaths occur due to $\mathrm{BC}$ in the developed countries. BC may develop due to unhealthy lifestyles, physical inactivity, obesity, late menopause, alcohol consumption, long time fertility with menarche in early ages and menopause in old ages, use of preventive pregnancy hormones, and having no children. ${ }^{16,17}$ TNBC is a rare subtype of BC, which lacks the expression of ER, PR, and HER2 receptors. These tumors are low-differentiated carcinomas, belonging to the basal-like subgroup, and characterized by frequent local recurrence and organ metastases. ${ }^{6-14}$ They are associated with the occurrence of hereditary forms of BCs, which are caused by BRCA1 and sometimes by BRCA2 gene mutations. ${ }^{6}$ In the present study, the clinicopathological features and metastatic pattern of TNBC were analyzed in 152 female patients with TNBC to determine the relationship between these features and TNBC. In our study, only $17 \%$ of BC patients were diagnosed with TNBC. Similarly, in a study by Dogra et al., 18\% of BC patients were diagnosed with TNBC. However, $39.94 \%$ and $34.4 \%$ of BC patients were diagnosed with TNBC in studies conducted by Sharma et al. and Nabi et al.

Compared with non- TNBC forms of BC, TNBC is considered an illness of young age, which is diagnosed at an advanced stage with a large size tumor. ${ }^{6} \mathrm{BC}$ is diagnosed at younger age and at a more advanced stage in Saudi patients than in other country . In our study, the mean (SD) age of the patients at the time of diagnosis was $48.82( \pm 12.034)$ years, which is similar to those reported by Alzahrani et al. (51 years) and Negi et al. (49.6 years). ${ }^{18-19}$ In our study, the majority (32.9\%) of the patients were in Group 3 (42-50 years), which is similar to that reported by Giri et al. (approximately $70 \%$ of the patients were $40-50$ years old).$^{20}$ By contrast, Alzahrani et al. and Negi et al. reported that maximum patients were $>50$ years old. ${ }^{18-19}$ These differences may be due to the fact that our study patients received medical care at an early age because of the availability of tertiary health care, leading to appropriate diagnosis and treatment. No significant correlation was found between age, 
tumor size, metastasis, axillary lymph node status, BMI and tumor size, grade.

The mean (SD) BMI in our study was found to be $29.3( \pm 6.43)$, and the majority $(42.1 \%)$ of the patients were obese followed by overweight $(28.9 \%)$. This confirmed that obesity with unhealthy lifestyle and no physical activity is a significant factor associated with the development of BC. Alzahrani et al. obtained similar results (mean [SD] BMI: 29.59 [ \pm 6.8$]$ ) and reported that most (76.9\%) of the patients were overweight/obese. ${ }^{18}$ Weight gain is a persistent and common issue in $\mathrm{BC}$ patients, especially in young patients and patients. ${ }^{21}$ Several studies have reported that weight gain after diagnosis either increases the risk of cancer. ${ }^{22-25}$ Further, BMI of non-Saudi patients was higher than that of Saudi patients, which may be due to the fact that non- Saudi patients were less active and typically lived a sedentary lifestyle compared with Saudi patients. Additionally, the highest mean BMI (30.85) was noted in Group 5 (>60 years) followed by that in Group 4 (50-60 years), which is in agreement with the previous statement.

The symptoms experienced by patients were breast pain, nipple discharge, palpable axillary lymph node, skin changes, and nipple changes. The most common location of malignant breast mass is the left upper outer quadrant. ${ }^{22,23}$ In our study, the most common symptoms were palpable axillary tail mass $(17.1 \%)$, breast pain (16.4\%), and nipple changes $(14.5 \%)$. Only $3.3 \%$ of the patients experienced nipple discharge in our study. Further, $9.9 \%$ of the patients had a family history of BC. Similarly, $16.7 \%$ of the TNBC patients had a family history of $\mathrm{BC}$ in the study by Sun et al. In addition, Haffty et al. reported that a large proportion of TNBC patients have a family history of BC. ${ }^{24}$

TNBC patients are prone to distant metastasis and local recurrence. TNBC may increase the risk of distant metastasis or local recurrence following the final diagnosis. TNBC recurrence pattern may differ from other biological subtypes of breast cancer. Additionally, one of the most characteristic sites of metastasis is the lung and brain. ${ }^{24-25}$ In our study, distant metastasis was observed in a significant number (33.6\%) of patients; non-Saudi patients exhibited a higher rate of metastasis $(39.3 \%)$ than Saudi patients $(25.4 \%)$. Bone metastasis was observed in $14.5 \%$ of the patients, liver metastasis in $15.8 \%$, lung metastasis in $22.4 \%$, brain metastasis in $3.3 \%$, and skin metastasis in $2 \%$. By contrast, in the study by Sun et al., the distant metastasis rate was $8.8 \%$; bone and visceral metastases were noted in $2.5 \%$ and $10.5 \%$ of the patients, respectively. ${ }^{24}$ Distant metastasis in TNBC may have organ-specific tendency, which may be associated with specific gene expression. Dent et al. reported that compared with non-TNBC patients, the incidence of distant metastasis increased significantly with increase in the risk of distant metastasis among TNBC patients. ${ }^{25}$ Further, BC recurrence 
was not noted in the majority $(79.6 \%)$ of the patients, the recurrence rate was the highest (27.3\%) in Group 1 (20-30 years) and the lowest in Group 5 ( $>60$ years). The recurrence rate reported by Sun et al. was low (6.7\%) and that by Sharma et al was high (45.6\%), with metastasis/local recurrence in TNBC patients.

\section{Conclusions:}

No significant correlation was found between age, tumor size, age and metastasis, age and axillary lymph node status, BMI and tumor size as well as BMI and tumor grade of TNBC patients. In addition, the metastatic and recurrence rates were high in our study. TNBC, an aggressive disease that is diagnosed in young individuals, was frequently diagnosed among middle aged individuals in our study. In addition, the majority of TNBC patients were obese and overweight, suggesting that weight of the patients is a significant factor associated with the development of TNBC. Further studies with large study population examining the influence of weight and age in TNBC prognosis are warranted. Further studies are required for the determination of significant correlations between these factors and TNBC on a global scale.

\section{Disclosure:}

The author declares no competing financial interest. Furthermore, the work was not supported by any agency.

\section{References:}

[1] Ghoncheh M, Pournamdar Z, Salehiniya H: Incidence and Mortality and Epidemiology of Breast Cancer in the World. Asian Pacific Journal of Cancer Prevention. 2016, 17:43-46. 10.7314/APJCP.2016.17.S3.43

[2] Saggu S, Rehman H, Abbas ZK, Ansari AA: Recent incidence and descriptive epidemiological survey of breast cancer in. Saudi Arabia. Saudi medical journal. 2015, 36:1176. 10.15537/smj.2015.10.12268

[3] Elkablawy MA, Albasry AM, Hussainy AS, Nouh MM, Alhujaily A: Molecular profiling of breast carcinoma in Almadinah, KSA: immunophenotyping and clinicopathological 
correlation. Asian Pac J Cancer Prev. 2015, 16:7819-7824. 10.7314/APJCP.2015.16.17.7819

[4] Navrátil J, Fabian P, Palácová M, Petráková K, Vyzula R, Svoboda M: Triple Negative Breast Cancer. Klinicka onkologie. Llin Onkol.2015;28(6):405-15.

[5] Kaplan HG, Malmgren JA: Impact of triple negative phenotype on breast cancer prognosis. Breast J. 2008, 14:456-463. 10.1111/j.1524-4741.2008.00622.X

[6] Lee JA, Kim KI, Bae JW, Jung YH, An H, Lee ES: Korean Breast Cancer Society Triple negative breast cancer in Korea-distinct biology with different impact of prognostic factors on survival. Breast Cancer Res Treat. 2010, 123:177-187. 10.1007/s10549-010-0998-5

[7] Dent R, Trudeau M, Pritchard KL, et al.: Triple-negative breast cancer: clinical features and patterns of recurrence. Clin Cancer Res. 2007, 13:4429-4434. 10.1158/1078-0432.CCR-06-3045

[8] Nishimura R, Arima N: Is triple negative a prognostic factor in breast cancer?. Breast Cancer. 2008, 15:303-308. 10.1007/s12282-008-0042-3

[9] Carey LA, Dees EC, Sawyer L, et al.: The triple negative paradox: primary tumor chemosensitivity of breast cancer subtypes. Clin Cancer Res. 2007, 13:2329-2334. 10.1158/1078-0432.CCR-06-1109

[10] Mersin H, Yildirim E, Berberoglu U, Gulben K: The prognostic importance of triple negative breast carcinoma. Breast. 2008, 17:341-346. 10.1016/j.breast.2007.11.031

[11] Liedke C, Mazouni C, Hess KR, et al.: Response to neoadjuvant therapy and long-term survival in patients with triple-negative breast cancer. J Clin Oncol. 2008, $26: 1275-1281 . \underline{10.1200 / \mathrm{JCO} .2007 .14 .4147}$

[12] Lin NU, Vanderplas A, Hughes ME, et al.: Clinicopathologic features, patterns of recurrence, and survival among women with triple-negative breast cancer in the National Comprehensive Cancer Network. Cancer. 2012, 10.1002/cncr.27581.

[13] Chlebowski RT: Nutrition and physical activity influence on breast cancer incidence and outcome. Breast 10.1016/j.breast.2013.07.006.

[14] Alzahrani W, Althoubaity F, Alsobhi D, et al.: Clinicopathological Features and Metastatic Pattern of Triple-positive Breast Cancer Among Female Patients at a Tertiary Care Hospital. Cureus. 2019, 11:12. 10.7759/cureus.6458

[15] Negi P, Kingsley PA, Jain K, Sachdeva J, Srivastava H, Marcus S, Pannu A: $\underline{\text { Survival of }}$ triple negative versus triple positive breast cancers: comparison and contrast. Asian Pac J Cancer. 2016, 17(8):3911-6 
[16] Giri R, Sahu S, Senapati U: Clinicopathological features of triple negative breast carcinoma-An experience from a tertiary care hospital. IOSR-JDMS. 2018, 10-12 20:1. 10.9790/0853-1708121012".

[17] Makari-Judson G, Braun B, Jerry DJ, Mertens WC: Weight gain following breast cancer diagnosis: Implication and proposed mechanisms. World J Clin Oncol. 2014, 5:272-282. 10-5306/wjco.v5.i3.272

[18] Nichols HB, Trentham-Dietz A, Egan KM, et al.: Body mass index before and after breast cancer diagnosis: associations with all-cause, breast cancer, and cardiovascular disease mortality. Cancer Epidemiol Biomarkers Prev. 2009, 18:1403-1409. 10.1158/1055-9965.EPI-08-1094

[19] Caan BJ, Kwan ML, Hartzell G, Castillo A, Slattery ML, Sternfeld B, Weltzien E: Pre-diagnosis body mass index, post-diagnosis weight change, and prognosis among women with early stage breast cancer. Cancer Causes Control. 2008, 19:1319-1328.

[20] Sun W, Li C, Liu M, Liu W, Yang C, Cai LI: Prognostic analysis of triple-negative breast cancer patients treated with adjuvant chemotherapy of fluorouracil, epirubicin and cyclophosphamide. Oncology letters. 2016, 11:2320-2326. 10.3892/oI.2016.4176

[21] Weidhaas J, Harris L, Hait W, Toppmeyer D: Locoregional relapse and distant metastasis in conservatively managed triple negative early-stage breast cancer. J Clin Oncol. 2006, 24:5652-5657. 10.1200/JCO.2006.06.5664

[22] Rakha EA, El Sayed ME, Green AR, Lee AH, Robertson JF, Ellis IO: Prognostic markers in triple negative breast cancer. Cancer. 2007, 109:25-32. 10.1002/cncr.22381

[23] Rodríguez-Pinilla SM, Sarrió D, Honrado E, et al.: Vimentin and laminin expression is associated with basal-like phenotype in both sporadic and BRCA1-associated breast carcinomas. J Clin Pathol. 2007, 60:1006-1012. 10.1136/JCP.2006.042143

[24] Niwińska A, Murawska M, Pogoda K: Breast cancer brain metastasis: differences in survival depending on biological subtype, RPA RTOG prognostic class and systemic treatment after whole brain radiotherapy (WBRT). Ann Oncol. 2010, 21:942-948. 10.1200/jco.2008.26.15

[25] Dent R, Trudeau M, Pritchard KI, et al.: Reduced risk of axillary lymphatic spread in triple-negative breast cancer. Clin Cancer Res. 2015,49:229-236. 